\title{
Large scale elections by coordinating electoral colleges
}

\author{
A. Riera, J. Borrell, J. Rifà \\ Dept. d'Informàtica, Universitat Autònoma de Barcelona \\ Edifici C - 08193 Bellaterra - Catalonia (Spain) \\ Tel: + 3435811777 - Fax: + 3435813033 \\ E-mail: andreu@ccd.uab.es
}

\begin{abstract}
In this paper we propose a truly very large scale voting scheme useful in national and international elections. We offer an implementable solution in which the voting authority is distributed among several voting centres. Each centre acts as an electoral college, carrying out a local scale ballot. The global tally is therefore computed by joining all partial tallies.

Our proposal consists on a hierarchical arrangement of the voting centres, strongly based on the use of X.500 Directory Service. Our scheme allows to solve many coordination problems: distribution of voters, coordination of the opening and closing times, management of the electoral roll, distribution of public keys, distribution of partial tallies, and computation of the global tally.
\end{abstract}

\section{Keywords}

Electronic voting schemes, X.500 Directory Service, wide area computer networks

\section{INTRODUCTION}

The first cryptographic scheme for secure electronic voting was introduced by Chaum (Chaum 1981). Many different proposals (over twenty) have appeared subsequently. Most of them consist in a set of cryptographic tools and protocols that are used together to fulfil the commonly accepted security requirements (Fujioka, Okamoto and Ohta 1992):

- Completeness: All valid votes are counted correctly.

- Soundness: The dishonest voter cannot disrupt the voting.

- Privacy: All votes must be secret.

- Unreusability: No voter can vote twice.

- Eligibility: No one who is not allowed to vote can vote. 
- Fairness: The counting of ballots does not affect the voting.

- Verifiability: No one can falsify the result of the voting.

However, most of the proposals are designed without caring about the complexity of their possible real implementations. Consequently, the computation and communication costs usually prevent the development of a practical product.

Only four voting schemes have been implemented over a computer network. (Hassler and Posch 1995) and (Cramer and Cytron 1996) have been developed as simplifications of theoretical proposals - (Nurmi, Salomaa and Santean 1991) and (Fujioka, Okamoto and Ohta 1992), respectively-. However, such simplifications break some security requirements. A dishonest voter is allowed to disrupt the entire voting in (Hassler and Posch 1995), and a dishonest ballot collecting authority may break the fairness condition in (Cramer and Cytron 1996). In contrast, the proposals in (Hwang 1996) and (Borrell and Rifâ 1996) were specially designed with practical implementations in mind. Furthermore, they adopt a conventional approach which has a better chance of gaining social acceptance. For example, (Borrell and Rifâ 1996) uses an electoral board to administer the ballot.

Nevertheless, the designs of the four implemented schemes assume restricted environments involving a single voting centre. The number of voters is not expected to be large and the implementations are typically operated on a LAN. A single voting centre is clearly not enough when the number of voters is potentially very large. Even using simple protocols (as that proposed in (Fujioka, Okamoto and Ohta 1992)) a number of voting centres should be considered. Only (Hassler and Posch 1995) gives little directions on how to operate a hierarchy of voting centres, as an ampliation of the scheme, but without making more specific.

In this paper we present the design of an electronic voting scheme assuming a very large number of voters. It may be operated over a WAN or a group of interconnected networks (ideally the whole Internet). Our proposal is based on the concurrent operation of a set of electoral colleges (ECs), each of them carrying out a local ballot. We have adopted (Borrell and Rifâ 1996) as the local voting scheme operated by the electoral colleges because it is specially concerned with the security requirements offered by the scheme.

The coordination of the set of electoral colleges poses several open questions that must be solved: distribution of voters, coordination of the opening and closing times, management of the electoral roll, distribution of public keys, distribution of partial tallies, and computation of the global tally. Our scheme offers mechanisms to solve these questions through a hierarchical arrangement of voting centres. We make extensive use of X.500 Directory Service (CCITT 1989a) to support the management of the whole system. We have adopted simplicity and efficiency as major requirements of our system, keeping in mind that the final goal is to practically implement the scheme. 
The rest of the paper is structured as follows: In section 2 we show the overview of our model. Section 3 is concerned about the elaboration and use of the electoral roll by using X.500. Section 4 shows the policies related to key generation and key distribution procedures. Section 5 and 6 are devoted to the computation of the global tally from all partial results and its divulgation, respectively. Since all previous'sections suppose an election that involves only one nation, section 7 discusses several questions that arise when an international voting has to be performed. Finally, section 8 contains the conclusions of the work, analyzing the security offered by the model.

\section{OVERVIEW OF THE MODEL}

The voting scheme presented in (Borrell and Rifa 1996) operates well in a restricted environment with a small comunity of voters, typically a LAN. However, it is not suitable for a large scale election. The ballot box and the electoral roll files could grow to unmanageable proportions. Even worst, the voting centre would easily become a bottleneck.

Clearly, in a wide area environment with a large number of users, voters must be distributed among many ECs. Such collecting centres should be operated concurrently, each caring only of a small number of voters. However, such point of view requires some mechanisms to assure the coordination of all ECs. It must be guaranteed, for instance, that a particular voter can only cast his/her vote to a unique EC. Moreover, this approach ends up with a number of partial tallies which should be joined. The protocols used to join the tallies must guarantee the security requirements of the ballot.

To solve these problems, we use a nation-wide hierarchical model in which the vertices represent different voting authorities. We call such hierarchy the voting tree (see Figure 1). The hierarchical arrangement is an effective way to distribute the voting authority among the members of the community. Moreover, the task of joining partial countings is extremely eased.

The leaves of the voting tree represent the ECs. Every EC carries out an independent ballot within a reduced group of voters. Intermediate nodes act as Counting Centres (CCs). Their main task is to join partial tallies from the nodes belonging to their corresponding subtrees. The outcomes are passed up through the tree until they reach the root, which performs the computation of the global nation-wide tally and publishes it. This last entity is not only a counting centre, but it also maintains the electoral roll and does some administrative tasks related to the elections. It is also responsible for publishing the valid voting options before the voting is initiated. We call it the National Voting Authority (NVA). The NVA is unique within its country and it has a permanent nature. It is intended to be the electronic version of the official organization that manages the electoral roll in conventional elections. 


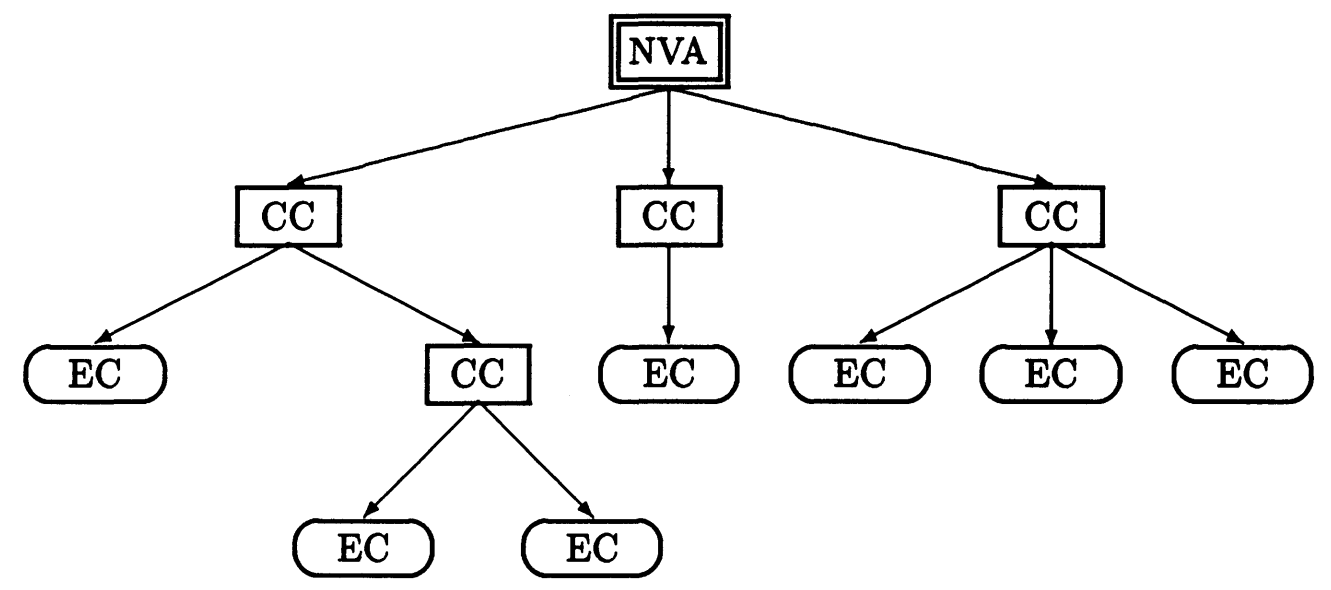

Figure 1 Hierarchical arrangement of voting authorities.

In Figure 1, we show an example of hierarchical arrangement of NVA, CCs and ECs. Any number of intermediate levels is possible. However, in order to simplify the discussions, in the rest of the paper we will suppose the voting tree has three levels: the root (NVA), an intermediate level (CCs), and the leaves (ECs).

\section{MANAGEMENT OF THE ELECTORAL ROLL}

In any election, the electoral roll must be used by the voting authority to determine whether a user is authorized or not to cast a vote. It can keep also a record of the voters who have already voted, to preserve the property of unreusability. Electronic voting schemes based on a single centre may use a locally stored file as electoral roll. In such case, any file format can be used. However, in a large scale scheme involving many voting centres, the electoral roll must be distributed and widely available on a commonly accepted format.

We plan to base the electoral roll on the CCITT X.500 Directory Service (CCITT 1989a). The main reasons are:

1. X.500 is an independent and totally distributed service which can be accessed from any point in the network. ECs do not need to care about it.

2. X.500 is standardized and therefore the uniformity of the operations on the Directory (consults, modifications) can be assured.

3. Access to the entries can be secured through strong authentication mechanisms (as described in X.509 (CCITT 1989b)). 
We suggest the creation of a subtree on the X.500 Directory Information Tree (DIT), which should be used only for voting purposes. The NVA acts as the authority responsible for the allocation of names within this subtree (i.e., the naming authority). The shape of this subtree is determined by the naming structure chosen by the NVA. This structure reflects the relationships described in the voting tree introduced in last section. Therefore, the NVA must be represented by the root entry. Its immediately subordinate entries represent the CCs. Next subordinates represent ECs. Finally, leaves represent voters and, therefore, the electoral roll. The immediate superior of the NVA's entry should be the entry representing the country involved in the election. Figure 2 shows an example of a voting subtree on the DIT.

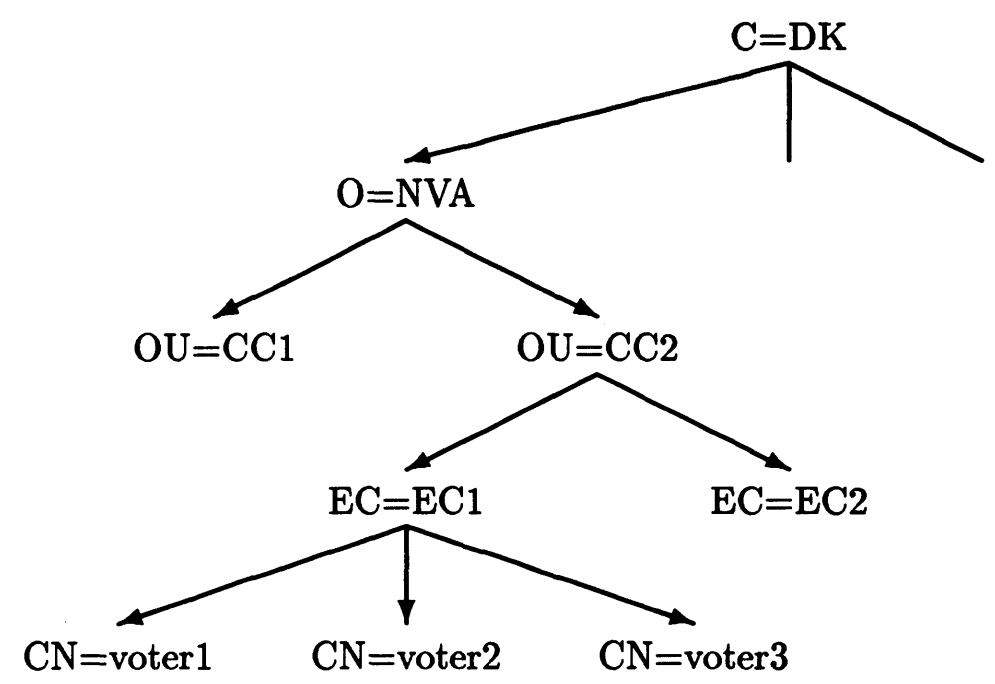

Figure 2 Voting subtree on the Directory Information Tree.

To create an independent subtree for the voting has the following advantages:

1. Duplicates are prevented if the naming structure of the subtree is carefully managed by the NVA. Each voter is assigned to only one EC only once. Note that every person may have different Distinguished Names disseminated across the whole DIT. Therefore, if already existing individual entries were used, mechanisms to solve the ambiguity had to be devised.

2. The shape of the subtree itself contains information about the hierachical relationship between the voting entities. This allows, for instance, to know which CC corresponds to a certain EC, just by examining the EC's Distinguiished Name. 
Concerning the electoral roll, there is besides a non-technical question that must be solved. It is related to the distribution of voters among the set of ECs. There are several alternatives to approach the problem. Each of them responds to a certain policy. It could be convenient, for example, to distribute voters on a territorial basis. In this way, each voter may be assigned to an EC depending on his/her locality.

In our model, we suppose that voters are users of a particular computer system that, in turn, is part of a local area network or group of interconnected local area networks (e.g., a company or university network). Such LAN environment is usually managed by an administrator. We assume every LAN will operate its own EC. The distribution of the software, tools and documentation required to operate the ECs is a task of the NVA. Letting voters cast their vote in their own LAN implies high efficiency at voting time. In addition, it is minimally intrusive to voters' normal behavior.

\section{X.500 Object Entries}

The NVA is represented in the Directory by an object of class organization + strongAuthenticationUser. The object class strongAuthenticationUser provides the attribute UserCertificate. The need for certificates will be discussed in section 4. In addition, the NVA's entry should support an additional attribute specific for our purposes: talliesEncryptionKey. The aim of this attribute will be explained in section 5. The ASN.1 definition of the Tallies Encryption Key is:

$$
\begin{aligned}
& \text { talliesEncryptionKey }::= \text { ATTRIBUTE } \\
& \text { WITH ATTRIBUTE-SYNTAX } \\
& \text { SignedKey }
\end{aligned}
$$

\section{SignedKey ::= SIGNED SEQUENCE \{ votingDate UTCTime subjectPublicKeyInfo SubjectPublicKeyInfo \}}

The entry of the NVA must be protected against creation and deletion of new components, updates, and naming modifications. Suck kind of access must be granted only to the NVA, after the proper authentication using cryptographically derived credentials (digital signatures). Detect, compare and read access to the attributes of the entry must be granted to anyone.

CCs are represented in the Directory by objects of class organizationalUnit + strongAuthenticationUser. The access rights of their entries follow the same phylosophy as that of the entry of the NVA. Only the CC represented by a particular entry is allowed to do writing operations on the attributes. The exception is the naming access category, which should be granted only to the NVA.

ECs are represented in the Directory by objects of class electoralCollege + strongAuthenticationUser. electoralCollege is a newly defined class with the following ASN.1 definition: 


\section{KEY MANAGEMENT}

In any cryptographic protocol involving public key cryptography, communicating peers must have knowledge of their partners' public keys. In this way, anyone is able to encrypt messages to every recipient and to verify any of their signatures. In our large scale voting model, asymmetric key pairs must be generated for each voter, each EC, each CC and the NVA. Public components must be properly certified by an independent infrastructure of trusted certification authorities (CAs). The X.500 Directory acts as a repository for the certificates.

The key generation and key certification procedures must follow different policies depending on whether the subject is the NVA, a CC, an EC or a voter.

The NVA must generate its own keys rather than allowing an independent $\mathrm{CA}$ to do it. The reason is the sensitiviness of such keys. However, the public key must be sent to a CA wich issues the corresponding certificate. The keys of the NVA do not have to be generated for each election but they may be used for several years (until the certificate expires). The certificate has to be published on the X.500 entry of the NVA.

The NVA generates also the asymmetric key pair formed by the Tallies Encryption Key and the Tallies Decryption Key. These keys are used when joining partial tallies (see section 5). The Tallies Encryption Key is not certified by any independent CA but it is rather directly signed by the NVA and placed on its X.500 entry. The Tallies Decryption Key must be stored in a safe local environment.

In contrast with the NVA, CCs are constituted just before each voting takes place. They may even be operated by different people each time. This suggests a new key pair must be generated for each ballot. As happens with the NVA, every CC generates its own keys and sends the public component to a certain $\mathrm{CA}$ which issues the certificate. After that, the certificate is published on the X.500 entry of the CC.

Every EC is operated by an electoral board of non-technical members. To guarantee the quality of the process of key generation, ECs' keys are directly generated by a CA. Note that ECs manage only a small number of voters and this makes their keys less sensitive than those of the CCs or the NVA.

Every CC orders to a certain CA the generation and certification of key pairs for all ECs which are under it on the voting tree. ECs must receive their key pairs by out of band means (at least the private component) from the CA. For instance, the members of the electoral board can physically pick up the keys at CA's site. Each EC is responsible for publishing the received certificate on its X.500 entry.

The case of voters is somewhat different from those of the other voting entities. The difference is that a voter, as a person, has many other roles not related with the elections. Consequently, it can be assumed that each voter will already own a public key, already certified and published on some entry on the X.500 Directory. 
Such certificate is already used by the user for any purpose (e.g., contract signing or electronic commerce). If a voter does not have any key pair yet, it must be generated before the voting takes place.

It can happen also that a voter has several key pairs, under different Distinguished Names. In such case, only one of them must be used for the voting. The chosen Distinguished Name must appear on the voter's entry at the electoral roll, as we have seen in section 3. In this way, a second access to X.500 allows to retrieve the proper public key.

The NVA is who must choose the identity (in practice, the public key) which will be used for the ballot among all those owned by a particular voter. The criteria used to choose the identity must be settled according to the established voting policies. There must be a complaining period, after the elaboration of the electoral roll and before the voting is initiated. Voters can force the NVA to make the proper changes and corrections on the electoral roll. Note that if users were allowed to directly choose their public key and send their decision to the NVA, communication overheads could be much greater.

\section{JOINING PARTIAL TALLIES}

The main task of the set of CCs is to gather partial tallies from the corresponding ECs. Tallies are joined, resulting wider partial countings. The process of joining partial tallies should guarantee the following properties:

1. No partial tallies must be public before the last EC is closed.

2. ECs must be able to verify the treatment their partial tallies have received in the corresponding CC.

3. At the phase of outcomes divulgation, it must be possible to elaborate statistics on every EC and every CC basis.

When the electoral board of a particular EC closes the local ballot, the control is passed to a counting process which elaborates the tally from the contents of the ballot box. As in conventional elections, once the ballot box has been opened and all the ballot papers counted, they are destroyed and only the final tally remains valid. The tally has a much simpler format than the ballot box. It is just a list containing pairs of the form (voting option, number of votes). To enforce the uniformity of all partial tallies, the NVA publishes (for instance, on the Web) the list of valid options before the ballot starts. Obviously, the list must be signed.

Any partial tally should be sent from the EC to the corresponding CC. Its simple format assures low communication overheads between ECs and CCs. Note that privacy of individual voters cannot be compromised at the CCs, since any personal information contained in the ballot box has been removed in the tally format. 
A major threat to the property of fairness appears when the opening and closing times of the ECs are not simultaneous. If the geographic area involved in the election is totally included in a single time zone, then all ECs open and close at the same time. However, to accomodate the major number of scenarios, we have supposed that ECs are scattered over several time zones. No partial results must be made public before all ECs have reached their closing time.

Our model guarantees the fairness of the voting by means of the Tallies Encryption Key (TEK). This public encryption key is generated by the NVA at the beginning of each election. Every EC retrieves the TEK from the X.500 entry of the NVA, and uses it to encrypt its partial tally. Therefore, CCs always receive partial tallies which are encrypted. The Tallies Decryption Key (TDK) is private and known only to the NVA. This assures nobody (but the NVA) is able to decrypt partial countings.

When an electoral board closes its local ballot, it sets the flag alreadyClosed in the EC's X.500 entry. In this way, the NVA can monitor the state of all ECs by checking these flags. When all ECs have closed, the NVA reveals the TDK to the CCs, thus allowing them to initiate the joining process.

Knowing the TDK, every CC can decrypt all partial tallies it has received, and elaborate a wider count with them. Such outcome must be sent to the NVA, which performs the ultimate joining of partial countings. In this way the global nation-wide tally is obtained. Note that the countings sent from the CCs to the NVA do not need to be encrypted. They cannot affect the voting because all ECs are already closed.

The authenticity and integrity of the data that is passed up through the voting tree must also be guaranteed. Before encrypting and sending any partial tally to a CC, ECs must sign a hash of the tally with their own private key. In the same way, CCs must sign their countings before sending them to the NVA. These signatures are universally verifiable by using the public keys held in the corresponding X.500 entries. Since public keys have been certified by independent trusted third parties, no voting authority can tamper the signatures on the tallies of lower entities. Moreover, every electoral board keeps a copy of its partial tally after it has been sent to the CC. In this way, it is possible to replace the tally if it is damaged or lost.

\section{DIVULGATION OF OUTCOMES}

To allow statistics on every EC basis, not only the global tally has to be divulgated, but all partial tallies and countings have to be published too. Publication of outcomes can be done on the Web due to its wide availability and scope.

The NVA and the CCs are responsible for the publication of countings. Every electoral board can verify the correct treatment of its partial tally by verifying its signature on the published outcomes. Nevertheless, anybody can do this task 
since all signatures are universally verifiable. Figure 3 shows the format of the list of outcomes published on the Web by the NVA.

\begin{tabular}{|l|l|}
\hline \multicolumn{2}{|c|}{ Date of the election } \\
\hline \multicolumn{2}{|c|}{ List of valid options } \\
\hline \multicolumn{2}{|c|}{ Global nation-wide tally } \\
\hline Partial counting 1 & Signature of CC 1 \\
\hline Partial counting 2 & Signature of CC 2 \\
\hline Partial counting 3 & Signature of CC 3 \\
\hline \multicolumn{2}{|c}{} \\
\hline \\
\hline
\end{tabular}

Figure 3 Divulgation of outcomes by the NVA.

CCs publish on the Web a document containing a list of the same characteristics of that presented in Figure 3. However, the field corresponding to the global tally is concerned only with the partial counting of that CC, and all partial outcomes are from ECs and not from CCs. Partial tallies are accompanied by the corresponding signature from the EC. The whole data structure is signed by the CC. Such document allows statistics on every EC basis. Note that CCs do not have to wait for any authorization before publishing their partial countings. No data has to be kept secret after the revelation of the TDK by the NVA.

\section{INTERNATIONAL ELECTIONS}

Preceding sections have supposed that the election affected only one country. If several countries are involved (for example in votings carried out by the States of the European Union) some specifications have to be considered. In particular, it has to be expected that each country would want to have absolute authority over its partial tally and its electoral roll. Therefore, it does not seem logical to convert each NVA on a CC (although it is possible from the technical point of view). 
The NVA of each country would still remain responsible for maintainig the corresponding voting subtree on X.500. It would also have to generate the TEK and TDK, and control their use within the country. Finally, it would elaborate the global tally for that country. In addition, an international voting authority (operated by an official organization like the European Commission) may act just as a counting centre to join all tallies from each country. We call such centre the International Counting Centre (ICC).

In each country the election takes place as it has been explained in previous sections. The only difference is that when all the ECs of a country have closed, the corresponding NVA does not reveal the TDK but it sends to the ICC a signed message indicating such condition. Once all the involved countries have informed the ICC about the closing of all ECs, the ICC authorizes the opening (decryption) of all partial tallies. This is achieved through the diffusion of a signed message from the ICC to the NVAs. The TDK for each country is then revealed by the corresponding NVA. Whenever a nation-wide tally is computed, the NVA has to send it to the ICC, signed but not encrypted. The signature removes any possibility of tampering. Divulgation of outcomes should follow the same scheme depicted in section 6 . This allows the elaboration of statistics also on every country basis.

To allow reliable verification of the signed messages interchanged between the ICC and the NVAs, their corresponding public keys must be known to each other. The ICC can retrieve NVAs' public keys from X.500. The distribution of ICC's public key is made also through certificates and X.500. In addition (due to its sensitiviness), out of band means may also be employed. This would not imply great overheads since the number of out of band communications would be very low (it would equal the number of countries involved in the election).

Note that elections inside very large companies can also involve several countries because of their multinational nature. However, such elections can follow the national model (i.e., without ICC) since only one policy is used in the whole community of voters.

\section{CONCLUSIONS}

In this paper we have presented a realistic proposal for a large scale electronic voting scheme. The ballot process is distributed through the concurrent operation of a number of Electoral Colleges. Each of them carries out a small scale voting, controlled by an electoral board. Partial tallies are joined by means of a hierarchical arrangement of Counting Centres. The figure of the National Voting Authority, at the root of the voting tree, is a permanent entity which cares about all administrative and preparative tasks related with the elections.

The model requires the following tools and elements: 
- X.500 Directory: The naming structure of the voting subtree reflects the hierarchical relationships between all voting entities. The electoral roll is maintained in voters' entries. Moreover, the Directory acts as repository of public key certificates.

- World Wide Web: It is used to publish the valid voting options before the election, and the outcomes after it.

- Certification infrastructure: A proper arrangement of Trusted Third Parties, independent from the election, issues the required public key certificates.

Assuming that the local voting scheme operated by each EC fulfils the commonly accepted security requirements (Fujioka, Okamoto and Ohta 1992), our proposal extends the security to wide area scope:

- Privacy: Every EC sends to the corresponding CC a partial tally, but not the ballot box in its original form (which is effectively erased when the local electoral board close the voting). The partial tally does not contain any information on individual ballot papers.

- Soundness, eligibility and unreusability: Secure access to X.500 is based on strong authentication mechanisms. They guarantee that each voter has a unique entry, and that only the authorized EC is allowed to modify the attribute voteAlreadyCast.

- Completeness and verifiability: Partial countings that are passed up through the voting tree are always protected by a digital signature. Such signature disables any corruption since all public keys are certified by an independent certification structure.

- Fairness: The TDK that allows to decrypt partial countings remains secret until all the electoral boards have closed. Only the NVA (which is a high-level entity) has to be trusted.

\section{ACKNOWLEDGEMENTS}

This work has been partially funded by the Spanish Government Commission CICYT, through a grant to the Combinatorics and Digital Communication Group.

\section{REFERENCES}

Borrell, J. and Rifâ, J. (1996) An Implementable Secure Voting Scheme. Computers \& Security, 15, 327-338.

Cramer, L.F. and Cytron, R.K. (1996) Design and Implementation of a Practical Security-Conscious Electronic Polling System. Technical Report WUCS96-02, Washington University, St. Louis. 
CCITT (1989) Blue Book, Volume VIII - Fascicle VIII.8, Data Communication Networks: Directory, Recommendations X.500-X.521, Geneva.

ISO-9594: Information Processing Systems - Open Systems Interconnection - The Directory.

CCITT (1989) Recommendation X.509. The Directory - Authentication Framework, Geneva.

See also ISO-9594-8.

Chaum, D. (1981) Untraceable Electronic Mail, Return Addresses and Digital Pseudonyms. Communications of the ACM, 24, 84-88.

Fujioka, A., Okamoto, T. and Ohta K. (1992) A Practical Secret Voting Scheme for Large Scale Elections. LNCS, Auscrypt '92, Springer Verlag.

Hassler, V. and Posch, R. (1995) A LAN Voting Protocol, in Proceedings of IFIP SEC '95, Ed. Chapman \& Hall, 176-189.

Hwang, J. (1996) A Conventional Approach to Secret Balloting in Computer Networks. Computers \& Security, 15, 249-263.

Nurmi, H., Salomaa, A. and Santean, L. (1991) Secret Ballot Elections in Computer Networks. Computers \& Security, 10, 553-560.

\section{BIOGRAPHY}

Andreu Riera, Joan Borrell and Josep Rifâ are members of the Combinatorics and Digital Communication Group at the Autonomous University of Barcelona.

Andreu Riera (Manresa, 1970) obtained the graduate degree in Computer Science in 1993 at the AUB. Since then he is working towards the Ph.D. degree in Computer Science in the field of Network Security.

Joan Borrell (Girona, 1965) obtained the graduate degree and the Ph.D. degree in Computer Science, in 1989 and 1996, respectively, at the AUB.

Josep Rifâ (Manlleu, 1951) obtained the graduate degree in Mathematics at the University of Barcelona in 1973. He obtained the Ph.D. degree in Computer Science in 1987 at the AUB. 